\section{Case Reports in Ophthalmology}

Case Rep Ophthalmol 2020;11:500-506

This article is licensed under the Creative Commons Attribution-NonCommercial 4.0 International License (CC BY-NC) (http://www.karger.com/Services/OpenAccessLicense). Usage and distribution for commercial purposes requires written permission.

\title{
Peripheral Ulcerative Keratitis in a Patient with Bilateral Scleritis: Medical and Surgical Management
}

\author{
June Artaechevarria Artieda ${ }^{a} \quad$ Nuria Estébanez-Corrales ${ }^{a}$ \\ Olga Sánchez-Pernaute ${ }^{b} \quad$ Nicolás Alejandre-Alba ${ }^{a}$ \\ aDepartment of Ophthalmology, Hospital Universitario Fundación Jiménez Díaz, \\ Madrid, Spain; 'b Department of Rheumatology, Hospital Universitario Fundación Jiménez \\ Díaz, Madrid, Spain
}

\section{Keywords}

Peripheral ulcerative keratitis · Scleritis · Lamellar keratoplasty · Superficial anterior lamellar keratoplasty · Amniotic membrane $\cdot$ Femtosecond laser

\begin{abstract}
Peripheral ulcerative keratitis (PUK) is a group of corneal disorders that cause peripheral corneal thinning, threatening globe integrity in advance stages. It is usually associated with systemic autoimmune diseases and management is based on local and systemic approaches. We present the case of a 47-year-old man with a previous history of bacterial keratitis in his left eye presenting with 1 month of bilateral ocular pain and redness. At examination, diffuse bilateral globe inflammation with paracentral corneal thinning in his left eye was observed. He was diagnosed with bilateral scleritis and PUK in his left eye. Workup for associated systemic autoimmune disease yielded negative results. The patient was started on pulses of intravenous methylprednisolone followed by oral prednisone failing to achieve sufficient control of the inflammatory syndrome. Subsequently, periodic intravenous cyclophosphamide was administered with a favorable response. A multilayer amniotic membrane graft was applied, but there was rapid melting with reabsorption of the tissue, resulting in extreme corneal thinning at the inferior paracentral cornea. A decentered $8.5-\mathrm{mm}$ superficial anterior lamellar keratoplasty (SALK) was then performed obtaining the donor graft with a femtosecond laser but performing manual trepanation in the recipient. At 12 months, visual and biomicroscopic measures do not
\end{abstract}




\section{Case Reports in Ophthalmology}

show deterioration and inflammation remains under control with oral azathioprine as maintenance regime. The management of PUK includes both systemic immunosuppression and tectonic procedures to preserve the globe integrity. Diverse surgical techniques have been attempted, but no definitive guidelines are available. Decentered large SALK is a simple technique that can yield acceptable visual results.

(C) 2020 The Author(s)

Published by S. Karger AG, Basel

\section{Introduction}

Peripheral ulcerative keratitis (PUK) is a group of inflammatory diseases that cause peripheral crescent-shaped corneal thinning. It is associated with autoimmune disorders such as rheumatoid arthritis, polyarteritis nodosa, systemic lupus erythematosus, granulomatosis with polyangiitis, or Churg-Strauss syndrome [1]. In 36\% of cases it is associated with scleritis [1].

In early stages, steroids and immunosuppressors are used to control the underlying condition and the ulcer is usually managed with antibiotics and intense lubrication. In advanced stages with extreme thinning or impending perforation, a surgical approach is needed in order to restore globe integrity and allow visual rehabilitation. Many surgical techniques have been described for peripheral perforations such as corneal wedge excision, targeted small-sized peripheral keratoplasty, large centered keratoplasty, or corneoscleral keratoplasty, but the most common technique is crescentic lamellar keratoplasty [2].

We report the case of a PUK in the context of bilateral scleritis without any known underlying systemic disease in which clinical management resulted challenging. On medical therapy, no response was obtained with oral steroids, necessitating alkylating agents. Surgically, a modified superficial anterior lamellar keratoplasty (SALK) was performed due to the extension and the localization of the ulcer.

\section{Case Report}

A 47-year-old Caucasian man consulted for severe pain and redness in both eyes for 1 month. His ophthalmologic background included a posttraumatic cicatricial ectropion of his left inferior eyelid that was reconstructed by a skin graft and a hard palate graft 3 years before; during the surgery, some glass fragments were extirped from the eyelid. He also had a corneal scar in the left eye with neovascularization, secondary to a corneal infiltrate caused by Moraxella catarrhalis 2 years before. The refractive error was $-2.5 \mathrm{D}$ sphere and $-0.75 \mathrm{D}$ cylinder in both eyes and he was using contact lenses $>8 \mathrm{~h}$ per day. He had no known systemic diseases.

At exploration his visual acuity was 0.9 in both eyes (decimals). Slit-lamp examination showed severe blepharitis and bilateral hyperemia with a corneal neovascularization area of $2 \mathrm{~mm}$ with mild stromal inflammation in the inferior part of the left eye. Neither endothelial deposits nor anterior chamber reaction were detected. Intraocular pressure was $19 \mathrm{~mm} \mathrm{Hg}$ in both eyes, and fundoscopy showed no alterations. The initial diagnosis was bilateral scleritis with an exudative inflammatory keratitis in the neovascularization area in the left eye. Conjunctival exudate samples were taken and resulted negative for bacterial and fungal growth. Treatment included dexamethasone drops every $6 \mathrm{~h}$ and moxifloxacin drops every $8 \mathrm{~h}$. The dexamethasone dose was reduced progressively during the next 7 months according to inflammation response. 


\section{Case Reports in Ophthalmology}

After the initiation of steroids and antibiotic drops the stromal keratitis improved. However, pain and hyperemia showed only mild response. Left inferior eyelash electrolysis was performed twice in the next months in order to avoid the risk of new epithelial defects caused by the contact of the eyelashes with the cornea.

Some months later, being under topical steroids every $24 \mathrm{~h}$, he consulted for worsening of the pain and hyperemia in both eyes. He was then diagnosed with bilateral chronic scleritis refractory to topical antibiotic and topical corticotherapy. Therefore, treatment with topical tacrolimus (Protopic ${ }^{\circledR}$ ) every $12 \mathrm{~h}$, and oral corticosteroids (prednisone $60 \mathrm{mg}$ per day for a week followed by 50, 40, 30, 20, 15, and $10 \mathrm{mg}$ weekly) were initiated. An extensive workup undertaken to exclude an accompanying autoimmune or infectious disease yielded negative results (which included a blood test with complete blood count, C-reactive protein, erythrocyte sedimentation rate, rheumatoid factor, antinuclear antibodies, antineutrophil cytoplasmic antibodies, complement, proteinogram, serology for infectious diseases, QuantiFERON, Rapid Plasma Reagin test, urine analysis, and chest X-ray). Two months later, a 1-mm-diameter thinned corneal ulcer was observed next to the neovascularization area, with $300 \mu \mathrm{m}$ of depth (Fig. 1). With the new diagnosis of PUK, a multilayer amniotic membrane graft was applied along with an intrastromal injection of antiangiogenic agents (bevacizumab). However, the amniotic membrane graft underwent rapid melting and subsequent reabsorption, leaving an amniotic membrane ceiling in the epithelization process devoid of underlying stroma with a total corneal thickness of $61 \mu \mathrm{m}$ (Fig. 2). Pulses of intravenous methylprednisolone (1 g daily per 3 consecutive days) were then administered, followed by oral prednisone $30 \mathrm{mg}$ per day, $100 \mathrm{mg}$ doxycycline every $12 \mathrm{~h}$ and vitamin C capsules $(1,000 \mathrm{mg}$ ) every $24 \mathrm{~h}$, as well as topical combination of tobramycin and dexamethasone every $6 \mathrm{~h}$.

At stabilization, a decentered 8.5-mm SALK of $300 \mu \mathrm{m}$ and a new amniotic membrane transplant were performed. The donor graft was prepared with femtosecond laser and the receptor cornea was manually trepanned. Postoperative treatment included tobramycin and dexamethasone drops every $6 \mathrm{~h}$ added to the previously mentioned systemic therapy. An important visual improvement was observed after the surgical procedure. In order to taper corticosteroids, the patient was put on monthly intravenous cyclophosphamide pulses $(500 \mathrm{mg}$ per infusion) under the surveillance of the Rheumatology Division, and the hyperemia in both eyes was found to definitely subside. After seven doses of cyclophosphamide, treatment was switched to azathioprine as maintenance therapy.

One year after the initiation of azathioprine, the patient has a best corrected visual acuity of 0.6 in his left eye and is being followed up every 2 months (Fig. 3). Signs and symptoms remain stable with oral azathioprine (50 mg every $12 \mathrm{~h}$ ) and topical dexamethasone once a day. The patient has shown good tolerance to the immunosuppressants and no extraocular manifestations have been observed to date.

\section{Discussion}

This case illustrates the complexity of the management of PUK, particularly when an underlying inflammatory condition such as scleritis coexists. Lack of good clinical practice-based guidelines for its approach makes decision-making especially difficult. In this patient, we could observe a favorable outcome with a modified surgical keratoplasty in association with energic systemic immunosuppression.

Overall, the goal of medical treatment is to reduce inflammation, to prevent superinfection, and to promote healing of the ulcer [1]. As regards local inflammation, topical steroids 


\section{Case Reports in Ophthalmology}

are most commonly used in association with topical antibiotics to avoid infection of the ulcer. Although steroids effectively reduce the localized inflammation in the cornea, they should be used with caution because these drugs inhibit new collagen synthesis and their application can thus result in a delay in epithelial healing. For the underlying inflammatory or autoimmune disease, systemic immunosuppression is usually done with pulses of methylprednisolone aimed at achieving a rapid response, followed by oral prednisone in a tapering schedule starting at $0.5 \mathrm{mg} / \mathrm{kg} /$ day. In nonresponding or severe cases, steroid-sparing agents such as antimetabolites, T cell inhibitors, alkylating agents, and biologic agents are indicated. In our case, cyclophosphamide was chosen based on the successful results reported in the literature, which had similar outcomes [3, 4].

In cases of extreme corneal thinning or impending perforation, the globe integrity needs to be preserved. In this context, many surgical techniques have been employed such as cyanoacrylate tissue adhesive, amniotic membrane grafts, bandage contact lenses, penetrating keratoplasties (PKs), lamellar grafts, adjacent conjunctival resections, and corneoscleral transplants $[2,5]$. In our case, the ulcer was first managed with a multilayer amniotic membrane graft due to the availability of this tissue in our center. This technique has been reported to be successful in many publications [6]. Notwithstanding, after the rapid melting and the reabsorption of the graft, it was decided to perform a more definitive procedure. Crescentshaped keratoplasty is the most recommended technique in PUK [2], but the paracentral situation of the ulcer in our patient precluded its use, since the border of the graft would have been placed near the visual axis. PK can be considered a fair therapeutic alternative in peripheral ulcerations, even though a high failure rate has been described in patients with recurrent melts [7]. Moreover, a graft survival of $20-40 \%$ at 6 months has been reported in PKs, requiring multiple grafts [7], while the risk of endothelial graft rejection implicated in PKs could be reduced with lamellar keratoplasties.

On the other hand, eccentric-shaped keratoplasties (biconvex, crescentic, round) are also reported to have good results in terms of visual acuity and not to induce astigmatism on the central corneal surface $[8,9]$, but these techniques could not be used in our case as they would have affected the visual axis. Considering all these facts, we decided for a decentered largediameter 8.5-mm SALK. The donor graft was shaped with femtosecond laser in order to improve its accuracy [10]. Trepanation of the receptor cornea was manually performed, since applanation and high intraocular pressure during laser application would have carried a high risk of damage in this patient and because of the need of performing perilimbal corneal cuts. We could show a satisfactory result using this modified SALK technique, which has been seldom reported. It should be noted that immediate postoperative visual acuity is not to be taken as a good outcome measure because it can be affected by suture-induced astigmatism. However, this fact does not alter visual acuity in the long term, as illustrated in our patient, who had a best corrected visual acuity of 0.6 at 12 months.

In summary, PUK should be regarded as a vision- and globe integrity-threatening disease. At presentation, a workup should be conducted to uncover an underlying systemic disease and intensive treatment should be applied for the accompanying condition. In mild cases, corneal thinning can be managed with topical steroids, antibiotics, and lubricants. On the other hand, a surgical approach is recommended in those cases with extreme corneal ulcerations or impending perforation. In ulcers with a relatively central disposition, as in the present case, large decentered SALK might be a good option, since it not only leaves the visual axis intact [11], but also because of the risk of endothelial graft rejection associated with PKs. In addition, they allow suturing in healthy corneal tissue, thereby avoiding melting areas. 


\section{Case Reports in Ophthalmology}

\begin{tabular}{l|l}
\hline DOI: $10.1159 / 000508325$ & (c) 2020 The Author(s). Published by S. Karger AG, Basel
\end{tabular} www.karger.com/cop

Artaechevarria Artieda et al.: Management in Peripheral Ulcerative Keratitis

\section{Statement of Ethics}

Written informed consent for publication of personal information and case details was obtained from the patient.

\section{Conflict of Interest Statement}

None of the authors have any conflict of interest to declare.

\section{Funding Sources}

The authors have no funding sources to disclose.

\section{Author Contributions}

J. Artaechevarria-Artieda made substantial contribution to the design of the work, the acquisition and interpretation of data, and the drafting of the work. N. Estébanez-Corrales made substantial contribution to the design of the work, the acquisition of data, and the drafting of the work. O. Sánchez-Pernaute made substantial contribution to the interpretation of data and revised the work critically for important intellectual content. N. Alejandre-Alba made substantial contribution to the design of the work as well as the acquisition and interpretation of data and revised the work critically for important intellectual content. All authors agree to be accountable for all aspects of the work.

\section{References}

1 American Academy of Ophthalmology. Stenz JM, Feldman BH, Woodward MA. Peripheral Ulcerative Keratitis. Available from: https://eyewiki.aao.org/peripheral_ulcerative_keratitis [cited March 11, 2015].

2 Lohchab M, Prakash G, Arora T, Maharana P, Jhanji V, Sharma N, et al. Surgical management of peripheral corneal thinning disorders. Surv Ophthalmol. 2019 Jan-Feb;64(1):67-78.

3 Clewes AR, Dawson JK, Kaye S, Bucknall RC. Peripheral ulcerative keratitis in rheumatoid arthritis: successful use of intravenous cyclophosphamide and comparison of clinical and serological characteristics. Ann Rheum Dis. 2005 Jun;64(6):961-2.

4 Ebrahimiadib N, Modjtahedi BS, Roohipoor R, Anesi SD, Foster CS. Successful Treatment Strategies in Granulomatosis With Polyangiitis-Associated Peripheral Ulcerative Keratitis. Cornea. 2016 Nov;35(11):1459-65.

5 Vislisel JM, Lenci L, Goins KM. Peripheral ulcerative keratitis (PUK). Available from: http://EyeRounds.org/cases/194-PUK.htm [cited August 25, 2014].

6 Solomon A, Meller D, Prabhasawat P, John T, Espana EM, Steuhl KP, et al. Amniotic membrane grafts for nontraumatic corneal perforations, descemetoceles, and deep ulcers. Ophthalmology. 2002 Apr;109(4):694703.

7 Jhanji V, Young AL, Mehta JS, Sharma N, Agarwal T, Vajpayee RB. Management of corneal perforation. Surv Ophthalmol. 2011 Nov-Dec;56(6):522-38.

8 Chern KC, Meisler DM, Wilson SE, Macsai MS, Krasney RH. Small-diameter, round, eccentric penetrating keratoplasties and corneal topographic correlation. Ophthalmology. 1997 Apr;104(4):643-7.

9 Parmar P, Salman A, Jesudasan CA. Visual outcome and corneal topography after eccentric "shaped" corneal grafts. Cornea. 2009 May;28(4):379-84.

10 Chamberlain WD. Femtosecond laser-assisted deep anterior lamellar keratoplasty. Curr Opin Ophthalmol. 2019 Jul;30(4):256-63. 
Case Reports in Ophthalmology
Case Rep Ophthalmol 2020;11:500-506

(c) 2020 The Author(s). Published by S. Karger AG, Basel www.karger.com/cop

Artaechevarria Artieda et al.: Management in Peripheral Ulcerative Keratitis

11 Feizi S, Javadi MA. Factors Predicting Refractive Outcomes After Deep Anterior Lamellar Keratoplasty in Keratoconus. Am J Ophthalmol. 2015 Oct;160(4):648-53.e2.

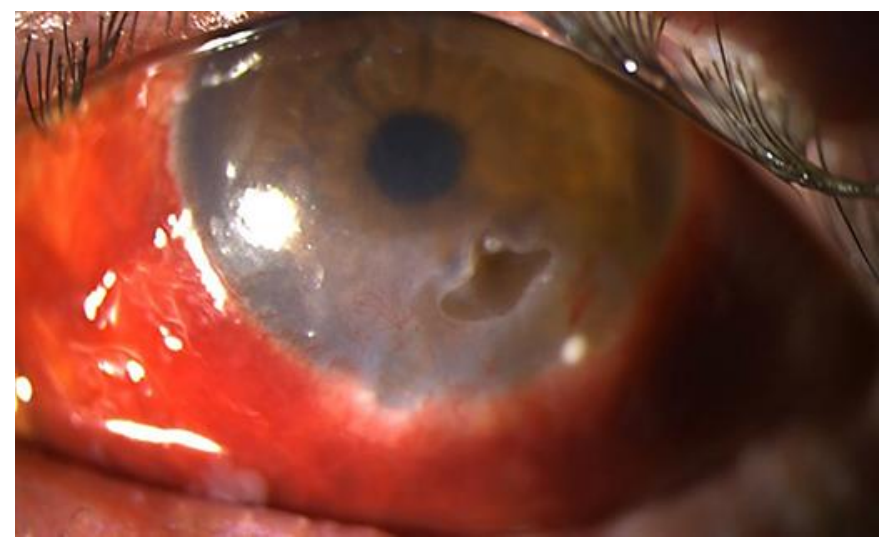

Fig. 1. Peripheral ulcerative keratitis in association with inferior neovascularization in the left eye with underlying bilateral scleritis.

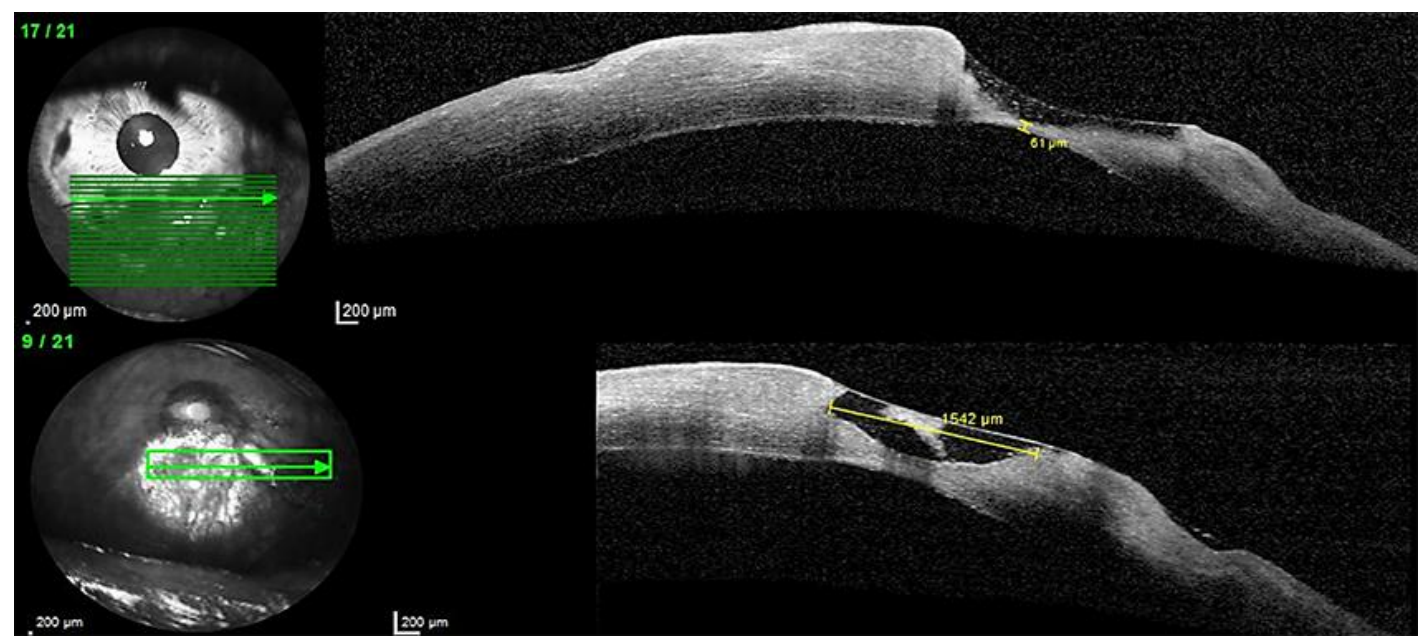

Fig. 2. Left eye AS-OCT (Cirrus HD-OCT, Zeiss Meditech, Germany) 1 week after multilayer amniotic membrane graft and an intrastromal injection of antiangiogenic agents (bevacizumab). Notice the rapid membrane reabsorption and corneal melting, with a total corneal thickness of $61 \mu \mathrm{m}$. 
Case Reports in Ophthalmology
Case Rep Ophthalmol 2020;11:500-506 DOI: $10.1159 / 000508325$

(c) 2020 The www.karger.com/cop

Artaechevarria Artieda et al.: Management in Peripheral Ulcerative Keratitis

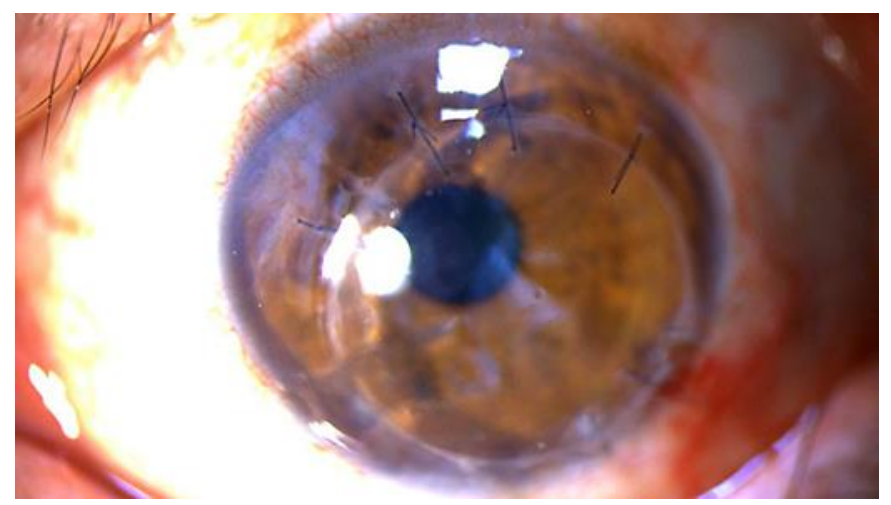

Fig. 3. An 8.5-mm decentered superficial anterior lamellar keratoplasty was performed in our patient. Stiches were removed as they became loose. 